\title{
Effects of anxiety, depression, and fatigue on quality of life in early esophageal cancer patients following endoscopic submucosal dissection
}

\author{
Yan Wu ${ }^{1}$, Yin Zhang ${ }^{2}$, Lili Zou ${ }^{3,4}$ \\ ${ }^{1}$ Department of Gastroenterology, the People's Hospital of Rugao, Rugao, China; ${ }^{2}$ Department of Digestive Diseases, the First People's Hospital of \\ Changzhou, Changzhou, China; ${ }^{3}$ Nursing Department, Wujin Hospital Affiliated with Jiangsu University, Changzhou, China; ${ }^{4}$ Nursing Department, \\ the Wujin Clinical College of Xuzhou Medical University, Changzhou, China \\ Contributions: (I) Conception and design: Y Wu, L Zou; (II) Administrative support: L Zou; (III) Provision of study materials and patients: Y Wu; \\ (IV) Collection and assembly of data: Y Wu; (V) Data analysis and interpretation: Y Wu, Y Zhang; (VI) Manuscript writing: All authors; (VII) Final \\ approval of manuscript: All authors. \\ Correspondence to: Lili Zou. Nursing Department, Wujin Hospital Affiliated with Jiangsu University, Changzhou 213002, China. Email: 778765909@qq.com.
}

Background: Emotional problems such as anxiety, depression, and fatigue may affect the quality of life $(\mathrm{QoL})$ of cancer patients. However, there are no related studies investigating the effects of emotional problems on the QoL of early esophageal cancer (EC) patients receiving endoscopic submucosal dissection (ESD).

Methods: This is a prospective observational study enrolling 75 early EC patients. Demographic and clinical characteristics of enrolled patients were recorded. QoL was assessed according to the European Organization for Research and Treatment of Cancer QoL (EORTC QLQ-C30). The Beck Anxiety Scale and the Beck Depression Scale were used to measure the severity of anxiety symptoms and depression intensity, respectively. Fatigue was assessed according to the symptom subscale of the EORTC QLQ-C30. Multivariable logistic regression models were constructed to determine the causes of emotional problems and linear regression models were employed to identify the independent risk factors for reduced QoL.

Results: Of the enrolled early EC patients, 21, 15, and seven had symptoms of mild, moderate and severe anxiety, respectively. Also, 15, 19, and two patients had symptoms of mild, moderate, and severe depression, respectively. In total, 27 patients frequently felt fatigue. The multivariable logistic regression model revealed that the financial capacity to pay for treatment had an important effect on the prevalence of anxiety and depression symptoms in enrolled patients (both $\mathrm{P}$ values $<0.001$ ). Age, gender, education status, and tumor size also played a role in emotional problems. Meanwhile, fatigue was found to be related only to age and gender. The mean score for QoL was 76.9 \pm 15.2. After adjusting for confounding factors using the multivariable linear regression model, moderate or severe symptoms of anxiety, depression, and fatigue, older age, and insufficient capacity to pay were identified as independent risk factors for reduced QoL.

Conclusions: Emotional distress is a significant problem for early EC patients receiving ESD treatment as it may affect their QoL.

Keywords: Anxiety; depression; fatigue; quality of life (QoL); endoscopic submucosal dissection (ESD); early esophageal cancer (early EC)

Submitted Jul 28, 2020. Accepted for publication Sep 25, 2020.

doi: $10.21037 /$ apm-20-1632

View this article at: http://dx.doi.org/10.21037/apm-20-1632 


\section{Introduction}

Esophageal cancer (EC) is among the most prevalent cancers of the upper digestive tract and was reported as the sixth most common cause of death due to malignant tumors worldwide (1). In China, EC has the sixth highest incidence rate among cancers, affecting an estimated 17.87 per 100,000 people, and a mortality rate of 13.68 per 100,000 people (2). EC has a poor prognosis, with a five-year survival rate of $20 \%$ (at most) (3). Surgical intervention can provide a relatively satisfying prognosis for patients with early or middle stage EC. However, esophagectomy for EC is often associated with a high incidence of complications that worsen the prognosis of patients (4).

The development of endoscopic submucosal dissection (ESD), which is now considered an acceptable treatment for early EC, has several advantages compared to esophagectomy including preservation of esophageal function, minimal invasiveness, less complications, lower cost, and shorter hospital stay (5). In addition, quality of life (QoL) following ESD is also considered as an important outcome for EC patients. Several studies have demonstrated that ESD provided a better postoperative QoL than surgery for early gastric and colorectal cancer patients $(6,7)$. However, many EC patients may suffer from physical and psychosocial problems due to cancer symptoms and side effects of treatment, which may dramatically impact their QoL. Previous studies have mainly focused on the effects of physical problems on QoL in EC patients $(8,9)$. Minimal attention has been given to the repercussions of emotional problems on the QoL of EC patients. Baudry et al. revealed that reducing the emotional distress of EC patients could improve their QoL either in the pre- or postoperative stage (10).

Patients enrolled in this study received surgical treatment. To our knowledge, there are no related studies investigating the effects of emotional factors on QoL in early EC patients. Hence, the present study was conducted to preliminarily investigate the QoL of early EC patients following ESD treatment and determine the effects of anxiety, depression, and fatigue on QoL. We present the following article in accordance with the STROBE reporting checklist (available at http://dx.doi.org/10.21037/apm-20-1632).

\section{Methods}

\section{Patient selection}

This was a prospective observational study to assess the
QoL of early EC patients. Early EC patients admitted to the People's Hospital of Rugao from December 2017 to January 2019 were enrolled in this study. Patients eligible for inclusion were older than 18 years of age, diagnosed as early EC (cT1aN0M0 or cT1bN0M0) by biopsy or imaging test, had no contraindications of ESD, received no previous surgical resection treatment and signed the written informed consent. Patients who refused to participate in the study or sign the written informed consent were excluded. The study was performed in compliance with the ethical principles outlined in the World Medical Association's Declaration of Helsinki (as revised in 2103) and was approved by the ethics committee of the People's Hospital of Rugao (No. 00201714).

\section{Data collection}

Demographic and clinical characteristics of patients were recorded following enrollment in the study. Recorded data included age, gender, education, marital status, employment status, capacity to pay for treatment, number of comorbidities, tumor stage, tumor location, tumor size, circumferential range, complete resection rate, and complications of ESD. All ESD procedures in this study were performed by skilled operators, in order to minimize the impacts of different operators on patients.

\section{Assessment of QoL}

QoL was assessed according to the European Organization for Research and Treatment of Cancer QoL (EORTC QLQ-C30) (11) within 3-6 months after the patients were discharged from hospital. EORTC QLQ-C30 is a selfreport scale specifically designed for cancer patients, and includes 30 items that can be divided into five functional scales (physical function, role function, emotional function, cognitive function, and social function) and one global health status scale. The total score in each scale ranges from 0 to 100, with a higher score indicating better QoL of patients. In this study, a total score of 75 was set as a cut-off; so a score $<75$ represented a poor QoL, while a score $>75$ represented a satisfying QoL.

\section{Assessment of anxiety, depression, and fatigue}

The Beck Anxiety Scale (BAS) was used to measure the severity of anxiety symptoms in enrolled patients (12). The BAS is a self-report scale and generally consists of 21 items, 


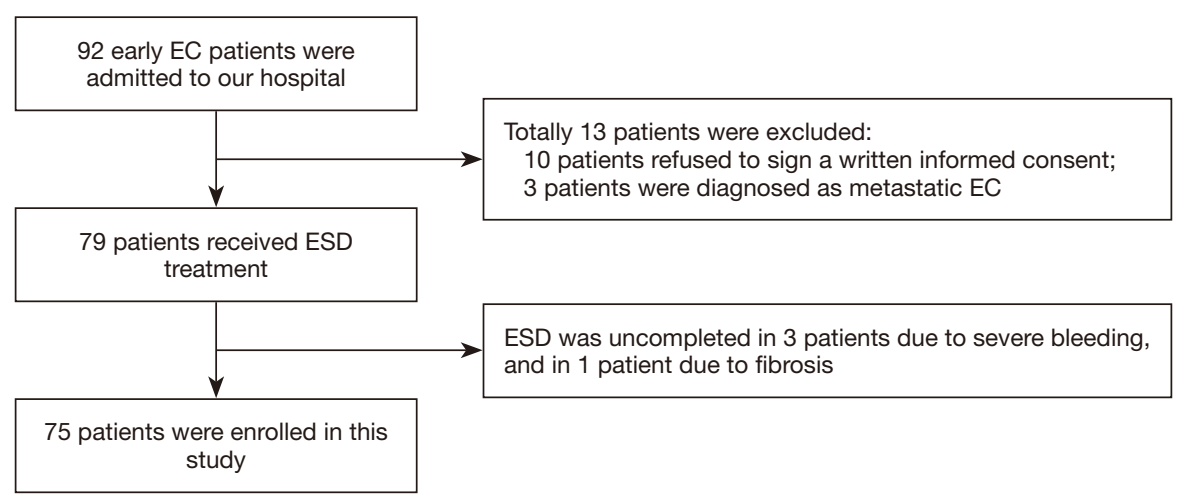

Figure 1 Flow chart showing the process of patient selection.

with each item being scored from 0 to 3 . Therefore, the total score of each patient ranges from 0 to 63 scores. In this case, a higher total score indicates more severe anxiety symptoms in patients. Our study defined anxiety symptoms as minimal if the total score was between 0 and 7 , mild if between 8 and 15, moderate if between 16 and 25, and as severe if between 26 and 63 .

The Beck Depression Scale (BDS) was then employed to assess the intensity of depression (13). Similar to the BAS, the BDS also contains 21 items, with each item being scored from 0 to 3 to obtain a total score ranging from 0 to 63. A total score of 0-9 indicated minimal depression, 10-18 indicated mild depression, 19-29 indicated moderate depression, and 30-63 indicated severe depression.

Fatigue was assessed according to the symptom subscale of the EORTC QLQ-C30. There were three items regarding physical strength in the symptom subscale, and each item could be scored from 0 to 3 scores. The total score of the three items were then transformed on a $0-100$ scale. In this study, a total score of $>75$ represented a poor condition of physical strength.

\section{Statistical analysis}

Statistical analyses were performed using SPSS (version 22.0, SPSS Inc., Chicago, IL, USA). Categorical variables were reported as numbers and percentages, and continuous variables were reported as means \pm standard deviations. Univariable and multivariable logistic regression models were constructed to determine the causes of three emotional problems, namely anxiety, depression, and fatigue. Furthermore, a linear regression model was employed to explore the impacts of the aforementioned emotional difficulties on the QoL of early EC patients undergoing
ESD. Some demographic and clinical characteristics of patients were adjusted in both multivariable regression models to reduce the bias of these data on the results. For all analyses, a $\mathrm{P}$ value $<0.05$ was considered statistically significant.

\section{Results}

In total, there were 92 early EC patients admitted to the hospital from December 2017 to January 2019. Of this initial cohort, 13 patients refused to participate in this study. ESD procedures were uncompleted in three patients due to severe bleeding, and in one patient due to fibrosis. Finally, 75 early EC patients were enrolled in this study (as shown in Figure 1). Demographic and clinical characteristics of enrolled patients are summarized in Table 1. The majority of patients were elderly, with the 60-70 years old cohort accounting for $48 \%$ of all included patients. There were 41 male and 34 female patients. Most patients were not well educated and only 12 patients had attended high school or above. Approximately $75 \%$ of the patients had a partner and 32 patients were employed at the time. Most importantly, roughly one third of patients had insufficient financial capacity to pay for treatment, and half of the patients suffered from at least two comorbidities.

In terms of cancer characteristics, only early EC patients were enrolled in this study. Of the 75 included patients, 53 were diagnosed as T1aN0M0 and 22 as T1bN0M0. Most tumors $(73.3 \%)$ were located in the middle of the esophagus. The tumor size was small $(<20 \mathrm{~mm})$ in 49 patients and relatively large $(\geq 20 \mathrm{~mm})$ in the remaining 26 patients. Tumors had invaded more than half of the circumference of the esophagus in 63 patients. 74 patients received a complete resection. 
Table 1 Demographic and clinical characteristics of enrolled patients $(\mathrm{n}=75)$

\begin{tabular}{|c|c|}
\hline Variables & $\mathrm{N}(\%)$ \\
\hline \multicolumn{2}{|l|}{ Age (years) } \\
\hline $40-50$ & $3(4.0)$ \\
\hline $50-60$ & $17(22.7)$ \\
\hline $60-70$ & $36(48.0)$ \\
\hline $70-80$ & $13(17.3)$ \\
\hline$>80$ & $6(8.0)$ \\
\hline \multicolumn{2}{|l|}{ Gender } \\
\hline Male & $41(54.7)$ \\
\hline Female & $34(45.3)$ \\
\hline \multicolumn{2}{|l|}{ Education } \\
\hline Primary school & $33(44.0)$ \\
\hline Middle school & $30(40.0)$ \\
\hline High school or above & $12(16.0)$ \\
\hline \multicolumn{2}{|l|}{ Marital status } \\
\hline Married & $57(76.0)$ \\
\hline Single or widowed & $18(24.0)$ \\
\hline \multicolumn{2}{|l|}{ Employed } \\
\hline Yes & $32(42.7)$ \\
\hline No & $43(57.3)$ \\
\hline \multicolumn{2}{|l|}{ Capacity to pay } \\
\hline Yes & $49(65.3)$ \\
\hline No & $26(34.7)$ \\
\hline \multicolumn{2}{|l|}{ Number of comorbidities } \\
\hline 0 & $21(28.0)$ \\
\hline 1 & $17(22.7)$ \\
\hline$\geq 2$ & $37(49.3)$ \\
\hline \multicolumn{2}{|l|}{ Tumor stage } \\
\hline T1aNOMO & $53(70.7)$ \\
\hline T1bNOM0 & $22(29.3)$ \\
\hline \multicolumn{2}{|l|}{ Tumor location } \\
\hline Upper & $7(9.3)$ \\
\hline Middle & $55(73.3)$ \\
\hline Lower & $13(17.3)$ \\
\hline
\end{tabular}

Table 1 (continued)
Table 1 (continued)

\begin{tabular}{lc}
\hline Variables & $\mathrm{N}(\%)$ \\
\hline Tumor size, mm & $49(65.3)$ \\
$<20$ & $26(34.7)$ \\
$\geq 20$ & \\
Circumferential range & $63(84.0)$ \\
$<1 / 2$ & $12(16.0)$ \\
$\geq 1 / 2$ & \\
Complete resection rate & $74(98.7)$ \\
Yes & $1(1.3)$ \\
No & \\
\hline
\end{tabular}

Table 2 Prevalence of emotional problems in enrolled EC patients $(\mathrm{n}=75)$

\begin{tabular}{lc}
\hline Variables & $\mathrm{N}(\%)$ \\
\hline Anxiety & $32(42.7)$ \\
Minimal & $21(28.0)$ \\
Mild & $15(20.0)$ \\
Moderate & $7(9.3)$ \\
Severe & \\
Depression & $39(52.0)$ \\
Minimal & $15(20.0)$ \\
Mild & $19(25.3)$ \\
Moderate & $2(2.7)$ \\
Severe & \\
Fatigue & $27(36.0)$ \\
Yes & $48(64.0)$ \\
No &
\end{tabular}

Emotional problems including anxiety, depression, and fatigue were assessed in all enrolled patients (Table 2). In general, 32 patients had symptoms of minimal anxiety, 21 patients had symptoms of mild anxiety, 15 patients had symptoms of moderate anxiety, and seven patients had symptoms of severe anxiety. With regards to depression, 39 patients had symptoms of minimal depression, 15 
patients had symptoms of mild depression, 19 patients had symptoms of moderate depression, and two patients had symptoms of severe depression. Furthermore, 27 patients frequently felt fatigued.

The causes of emotional distress in patients were analyzed using univariable and multivariable logistic regression models (as shown in Table 3). The multivariable logistic regression model revealed that older ( $\geq 60$ years) or female patients were more likely to suffer from moderate or severe anxiety than younger or male patients $(\mathrm{P}=0.045$ and 0.048 , respectively). The financial capacity to pay for treatment had a significant impact on the triggering of anxiety symptoms in the enrolled patients $(\mathrm{P}<0.001)$. Similarly, age, gender, and capacity to pay also played key roles in depression symptoms. Moreover, higher education level and smaller tumor size may be able to relieve symptoms of depression to some extent $(\mathrm{P}=0.048$ and 0.018 , respectively). Fatigue was found to be related only to age

Table 3 Demographic and clinical characteristics were associated with emotional problems in early EC patients according to the logistic regression model

\begin{tabular}{|c|c|c|c|c|c|c|c|c|c|c|c|c|}
\hline Variables & \multicolumn{4}{|c|}{ Anxiety } & \multicolumn{4}{|c|}{ Depression } & \multicolumn{4}{|c|}{ Fatigue } \\
\hline \multicolumn{13}{|l|}{ Age, years } \\
\hline \multicolumn{13}{|l|}{ Gender } \\
\hline Male & $33(62.3 \%)$ & $8(36.4 \%)$ & 0.044 & 0.048 & $35(64.8 \%)$ & $6(28.6 \%)$ & 0.006 & 0.022 & $32(66.7 \%)$ & $9(33.3 \%)$ & 0.007 & 0.026 \\
\hline Female & $20(27.7 \%)$ & $14(63.6 \%)$ & & & $19(35.2 \%)$ & $15(71.4 \%)$ & & & $16(33.3 \%)$ & $18(66.7 \%)$ & & \\
\hline High school or above & $11(20.8 \%)$ & $1(4.5 \%)$ & 0.375 & 0.496 & $11(20.4 \%)$ & $1(4.8 \%)$ & 0.654 & & $6(12.5 \%)$ & $6(22.2 \%)$ & 0.411 & \\
\hline \multicolumn{13}{|l|}{ Marital status } \\
\hline Married & $38(71.7 \%)$ & $19(86.4 \%)$ & 0.186 & & $38(70.4 \%)$ & $19(90.5 \%)$ & 0.083 & & $38(79.2 \%)$ & $19(70.4 \%)$ & 0.394 & \\
\hline Single or widowed & $15(28.3 \%)$ & $3(13.6 \%)$ & & & $16(29.6 \%)$ & 2 (9.5\%) & & & $10(20.8 \%)$ & $8(29.6 \%)$ & & \\
\hline \multicolumn{13}{|l|}{ Employed } \\
\hline Yes & $24(45.3 \%)$ & $8(36.4 \%)$ & 0.478 & & $22(40.7 \%)$ & $10(47.6 \%)$ & 0.867 & & $20(41.7 \%)$ & $12(44.4 \%)$ & 0.815 & \\
\hline No & $29(54.7 \%)$ & $14(63.6 \%)$ & & & $32(59.3 \%)$ & $11(52.4 \%)$ & & & $28(58.3 \%)$ & $15(55.6 \%)$ & & \\
\hline \multicolumn{13}{|l|}{ Capacity to pay } \\
\hline 1 & $10(18.9 \%)$ & $7(31.8 \%)$ & 0.788 & & $12(22.2 \%)$ & $5(23.8 \%)$ & 0.462 & & $9(18.8 \%)$ & 8 (29.6\%) & 0.578 & \\
\hline$\geq 2$ & 27 (50.9\%) & $10(45.5 \%)$ & 0.302 & & 28 (51.9\%) & 9 (42.9\%) & 0.692 & & 26 (54.2\%) & $11(40.7 \%)$ & 0.515 & \\
\hline
\end{tabular}

Table 3 (continued) 
Table 3 (continued)

\begin{tabular}{|c|c|c|c|c|c|c|c|c|c|c|c|}
\hline Variables & \multicolumn{4}{|c|}{ Anxiety } & \multicolumn{4}{|c|}{ Depression } & \multicolumn{3}{|c|}{ Fatigue } \\
\hline T1aN0M0 & $38(71.7 \%)$ & 15 (68.2\%) & 0.761 & & $39(72.2 \%)$ & $14(66.7 \%)$ & 0.636 & & 34 (70.8\%) & 19 (70.4\%) & 0.884 \\
\hline Upper & $6(11.3 \%)$ & $1(4.5 \%)$ & * & & $5(9.3 \%)$ & 2 (9.5\%) & * & & $5(10.4 \%)$ & $2(7.4 \%)$ & * \\
\hline Middle & 39 (73.6\%) & $16(72.7 \%)$ & 0.421 & & $41(75.9 \%)$ & $14(66.7 \%)$ & 0.659 & & 37 (77.1\%) & $18(66.7 \%)$ & 0.825 \\
\hline Lower & $8(15.1 \%)$ & $5(22.7 \%)$ & 0.279 & & $8(14.8 \%)$ & $5(23.8 \%)$ & 0.351 & & $6(12.5 \%)$ & 7 (25.9\%) & 0.287 \\
\hline \multicolumn{12}{|c|}{ Circumferential range } \\
\hline$<1 / 2$ & $44(83.0 \%)$ & 19 (86.4\%) & 0.720 & & $47(87.0 \%)$ & $16(76.2 \%)$ & 0.257 & & 42 (87.5\%) & $21(77.8 \%)$ & 0.276 \\
\hline$\geq 1 / 2$ & 9 (17.0\%) & $3(13.6 \%)$ & & & 7 (13.0\%) & 5 (23.8\%) & & & $6(12.5 \%)$ & $6(22.2 \%)$ & \\
\hline
\end{tabular}

${ }^{\mathrm{a}}$, the data were analyzed using a univariable logistic regression model; ${ }^{\mathrm{b}}$, the data were analyzed using a multivariable logistic regression model adjusting for the variables which showed significant difference in univariable analysis; * ${ }^{*}$ reference. EC, esophageal cancer.

Table 4 Summary of EORTC QLQ-C30 in enrolled EC patients

\begin{tabular}{ll}
\hline Variables & Mean \pm SD \\
\hline EORTC QLQ-C30 & \\
Physical function & $88.1 \pm 12.9$ \\
Role function & $85.5 \pm 16.8$ \\
Emotional function & $77.9 \pm 12.5$ \\
Cognitive function & $93.4 \pm 14.7$ \\
Social function & $91.0 \pm 17.2$ \\
Global health status & $76.9 \pm 15.2$ \\
\hline
\end{tabular}

EORTC QLQ-C30, European Organization for Research and Treatment of Cancer QoL; EC, esophageal cancer.

and gender.

The QoL of early EC patients was then assessed according to EORTC QLQ-C30. The mean scores of five functional scales and one global health status scale are summarized in Table 4. The mean scores in enrolled patients were as follows: physical function was $88.1 \pm 12.9$, role function was $85.5 \pm 16.8$, emotional function was $77.9 \pm 12.5$, cognitive function was $93.4 \pm 14.7$, and social function was
$91.0 \pm 17.2$. The mean score of global health status was 76.9 \pm 15.2 . Risk factors for reduced QoL were estimated according to univariable and multivariable linear regression models (shown in Table 5). Anxiety, depression, fatigue, age, gender, capacity to pay, and number of comorbidities were initially found to be significantly associated with QoL based on the results of the univariable linear regression analysis. However, after adjusting for confounding factors using a multivariable linear regression model, moderate or severe symptoms of anxiety, depression, and fatigue, older age, and insufficient capacity to pay for treatment were identified as independent risk factors for reduced QoL.

\section{Discussion}

To our knowledge, this is the first study to investigate the effects of anxiety, depression, and fatigue on QoL in early EC patients receiving ESD treatment. This study enrolled 75 patients from December 2017 to January 2019, and demonstrated that some demographic characteristics of patients including capacity to pay for treatment, age, gender, education status, and tumor size may cause emotional distress. Additionally, the prevalence of emotional problems, 
Table 5 Univariable and multivariable linear regression model estimating independent risk factors for reduced QoL in early EC patients

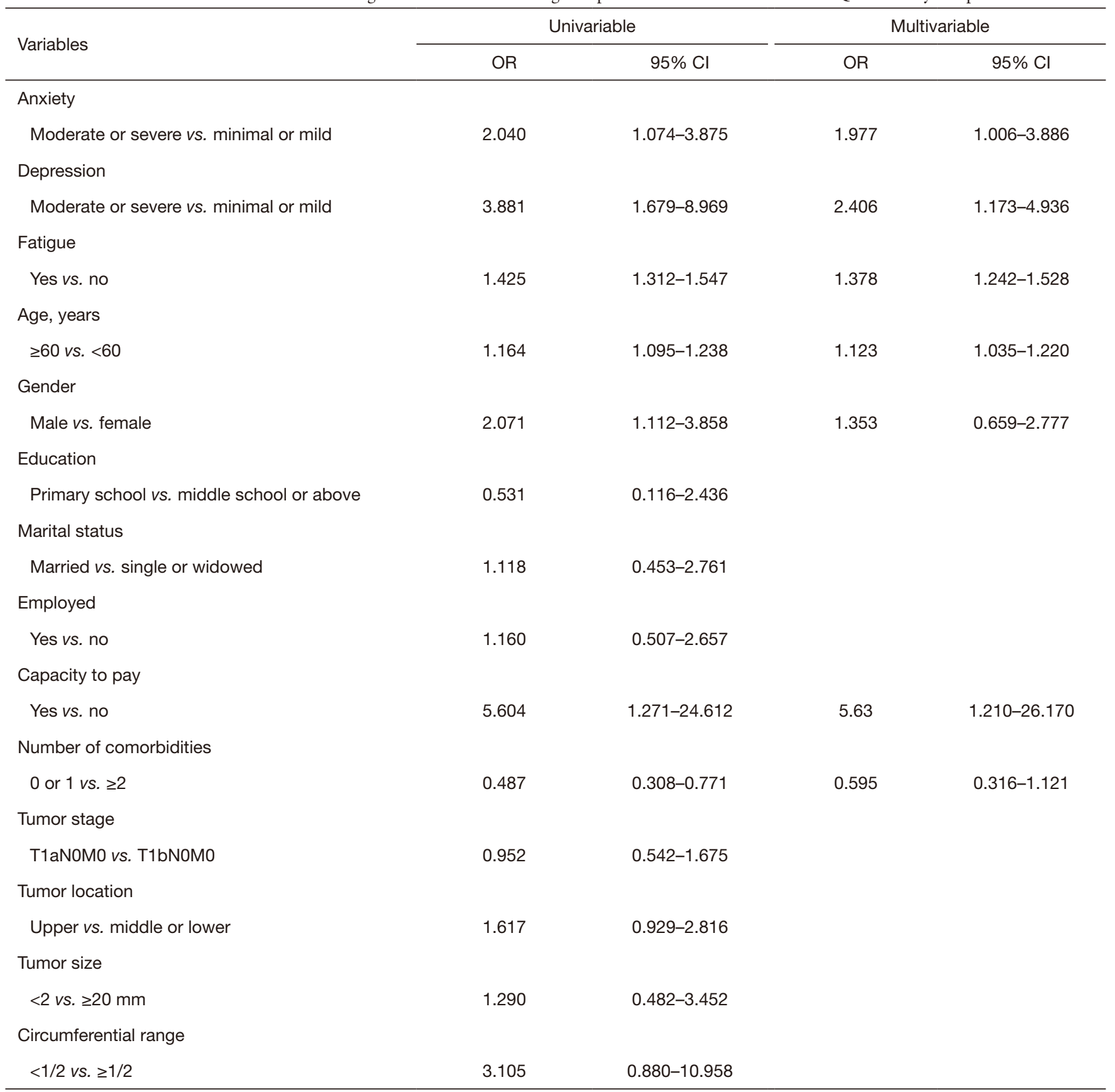

QoL, quality of life; EC, esophageal cancer.

such as anxiety, depression, and fatigue may reduce the QoL of early EC patients following ESD treatment.

The prevalence of anxiety (57.3\%) and depression (48.0\%) among the enrolled patients in the study were similar to previous studies involving cancer patients $(10,14)$. We further investigated the causes of emotional problems.
Several previous studies have reported that age and gender may be associated with the incidence of emotional distress in cancer patients $(15,16)$. Mols et al. reported that older and male colorectal cancer patients had considerably fewer symptoms of anxiety, yet exhibited more symptoms of depression (15). Moreover, Weiss Wiesel et al. found that 
anxiety decreased with age in older cancer patients receiving chemotherapy, while depression remained constant with age (17). However, our study found that the severity of both anxiety and depression symptoms decreased in older and male patients, which might be explained by cultural differences between diverse countries. In addition, early EC posed a relatively low psychological burden on patients compared with colorectal cancer.

It is understandable that an inability to pay the cost of treatment acted as a key cause of emotional distress. Several studies have also highlighted the important role of financial difficulties in the development of emotional problems in cancer patients $(18,19)$. Thus, measurements in the present study were taken to ensure that the rate of underdiagnosed psychiatric morbidities was reduced, especially for patients that are financially-burdened.

The most important finding of our study was the independent risk factors for reduced QoL in early EC patients. Depression was the major risk factor impacting QoL, especially functional status. Previous studies have shown that patients with depression typically experience a worse QoL compared with patients that do not suffer from depression (20,21). Our study obtained a similar result, indicating that the prevalence of depression significantly reduced the QoL score in early EC patients. Furthermore, we found that anxiety played a similar role to depression. The QoL of lung cancer patients was reported to be associated with both anxiety and depression (22). Bellali et al. proposed that early assessment of anxiety and depression may provide information and insight regarding vulnerable patients and guide the implementation of targeted treatment strategies (23). Fatigue is a common symptom in cancer patients and accounted for $36.0 \%$ in our study. Also, it itself was part of scale for assessing QoL. It has been shown that fatigue is associated with the presence of inflammation, as well as poor quality of sleep and QoL in patients with advanced cancer (24). The combination of multiple factors can induce fatigue in cancer patients. Our study demonstrated that fatigue was only related to age and gender considering the relatively low burden of cancer in the enrolled early EC patients.

Interestingly, the characteristics of the tumor had no significant relation to either emotional problems or QoL in early EC patients. In practice, most early EC patients do not require further chemotherapy after ESD treatment, and thus, ESD does not significantly increase the burden on patients. So, the reduced QoL in patients primarily resulted from emotional distress and financial difficulties.
Some limitations of this study should be noted. Firstly, only 75 early EC patients were enrolled in the study. This small sample size, coupled with the short study duration and single center study design hindered further analysis. Secondly, the majority of patients enrolled in this study were not well educated, which made it difficult to analyze the impact of education status on emotional problems and QoL. Thirdly, the incidence of esophageal stenosis after ESD may also be the main factor affecting the recovery and quality of life of patients. However, we didn't record the degree of stenosis in enrolled patients during the period of follow up.

In conclusion, ESD is considered an acceptable treatment for early EC and can provide better QoL outcomes than surgical procedures. Our prospective study verified the incidence of anxiety, depression, and fatigue in early EC patients following ESD treatment, which could reduce the QoL of EC patients. This finding emphasizes the importance of psychosocial health in patients receiving ESD treatment and highlights the need to strengthen psychological counseling in clinical practice.

\section{Acknowledgments}

Funding: This work was supported by the National Natural Science Foundation of China (81700575).

\section{Footnote}

Reporting Checklist: The authors have completed the STROBE reporting checklist. Available at http://dx.doi. org/10.21037/apm-20-1632

Data Sharing Statement: Available at http://dx.doi. org/10.21037/apm-20-1632

Conflicts of Interest: All authors have completed the ICMJE uniform disclosure form (available at http://dx.doi. org/10.21037/apm-20-1632). The authors have no conflicts of interest to declare.

Ethical Statement: The authors are accountable for all aspects of the work in ensuring that questions related to the accuracy or integrity of any part of the work are appropriately investigated and resolved. The study was performed in compliance with the ethical principles outlined in the World Medical Association's Declaration of Helsinki (as revised in 2013) and was approved by the 
ethics committee of the People's Hospital of Rugao (No. 00201714). Informed consent was taken from all the patients.

Open Access Statement: This is an Open Access article distributed in accordance with the Creative Commons Attribution-NonCommercial-NoDerivs 4.0 International License (CC BY-NC-ND 4.0), which permits the noncommercial replication and distribution of the article with the strict proviso that no changes or edits are made and the original work is properly cited (including links to both the formal publication through the relevant DOI and the license). See: https://creativecommons.org/licenses/by-nc-nd/4.0/.

\section{References}

1. Bray F, Ferlay J, Soerjomataram I, et al. Global cancer statistics 2018: GLOBOCAN estimates of incidence and mortality worldwide for 36 cancers in 185 countries. CA Cancer J Clin 2018;68:394-424.

2. Li H, Fang W, Yu Z, et al. Chinese expert consensus on mediastinal lymph node dissection in esophagectomy for esophageal cancer (2017 edition). J Thorac Dis 2018;10:2481-9.

3. Zhang $\mathrm{H}$, Yang $\mathrm{Y}$, Shang $\mathrm{Q}$, et al. Predictive value of preoperative weight loss on survival of elderly patients undergoing surgery for esophageal squamous cell carcinoma. Transl Cancer Res 2019;8:2752-8.

4. Darling GE. Quality of life in patients with esophageal cancer. Thorac Surg Clin 2013;23:569-75.

5. Kim SG, Ji SM, Lee NR, et al. Quality of Life after Endoscopic Submucosal Dissection for Early Gastric Cancer: A Prospective Multicenter Cohort Study. Gut Liver 2017;11:87-92.

6. Kim YI, Kim YA, Kim CG, et al. Serial intermediate-term quality of life comparison after endoscopic submucosal dissection versus surgery in early gastric cancer patients. Surg Endosc 2018;32:2114-22.

7. Nakamura F, Saito Y, Haruyama S, et al. Short-term Prospective Questionnaire Study of Early Postoperative Quality of Life After Colorectal Endoscopic Submucosal Dissection. Dig Dis Sci 2017;62:3325-35.

8. Yoshimura S, Mori K, Yamagata Y, et al. Quality of life after robot-assisted transmediastinal radical surgery for esophageal cancer. Surg Endosc 2018;32:2249-54.

9. Taioli E, Schwartz RM, Lieberman-Cribbin W, et al. Quality of Life after Open or Minimally Invasive Esophagectomy in Patients With Esophageal Cancer-A
Systematic Review. Semin Thorac Cardiovasc Surg 2017;29:377-90.

10. Baudry AS, Anota A, Mariette C, et al. The role of trait emotional intelligence in quality of life, anxiety and depression symptoms after surgery for esophageal or gastric cancer: A French national database FREGAT. Psychooncology 2019;28:799-806.

11. Aaronson NK, Ahmedzai S, Bergman B, et al. The European Organization for Research and Treatment of Cancer QLQ-C30: a quality-of-life instrument for use in international clinical trials in oncology. J Natl Cancer Inst 1993;85:365-76.

12. Beck AT, Epstein N, Brown G, et al. An inventory for measuring clinical anxiety: psychometric properties. J Consult Clin Psychol 1988;56:893-7.

13. İzci F, Sarsanov D, Erdogan Zİ, et al. Impact of Personality Traits, Anxiety, Depression and Hopelessness Levels on Quality of Life in the Patients with Breast Cancer. Eur J Breast Health. 2018;14:105-11.

14. Ohkura Y, Ichikura K, Shindoh J, et al. Relationship between psychological distress and health-related quality of life at each point of the treatment of esophageal cancer. Esophagus 2020;17:312-22.

15. Mols F, Schoormans D, de Hingh I, et al. Symptoms of anxiety and depression among colorectal cancer survivors from the population-based, longitudinal PROFILES Registry: Prevalence, predictors, and impact on quality of life. Cancer 2018;124:2621-8.

16. Polański J, Chabowski M, Chudiak A, et al. Intensity of Anxiety and Depression in Patients with Lung Cancer in Relation to Quality of Life. Adv Exp Med Biol 2018;1023:29-36.

17. Weiss Wiesel TR, Nelson CJ, Tew WP, et al. The relationship between age, anxiety, and depression in older adults with cancer. Psychooncology 2015;24:712-7.

18. Sudarisan SSP, Abraham B, George C. Prevalence, correlates of depression, and its impact on quality of life of cancer patients attending a palliative care setting in South India. Psychooncology 2019;28:1308-13.

19. Sharp L, O'Leary E, O'Ceilleachair A, et al. Financial Impact of Colorectal Cancer and Its Consequences: Associations Between Cancer-Related Financial Stress and Strain and Health-Related Quality of Life. Dis Colon Rectum 2018;61:27-35.

20. Calderon C, Carmona-Bayonas A, Hernandez R, et al. Effects of pessimism, depression, fatigue, and pain on functional health-related quality of life in patients with resected non-advanced breast cancer. Breast 
2019;44:108-12.

21. Fradelos EC, Papathanasiou IV, Veneti A, et al. Psychological Distress and Resilience in Women Diagnosed with Breast Cancer in Greece. Asian Pac J Cancer Prev 2017;18:2545-50.

22. Khue PM, Thom VT, Minh DQ, et al. Depression and Anxiety as Key Factors Associated With Quality of Life Among Lung Cancer Patients in Hai Phong, Vietnam. Front Psychiatry 2019;10:352.

23. Bellali T, Manomenidis G, Meramveliotaki E, et al. The impact of anxiety and depression in the quality of life

Cite this article as: Wu Y, Zhang Y, Zou L. Effects of anxiety, depression, and fatigue on quality of life in early esophageal cancer patients following endoscopic submucosal dissection. Ann Palliat Med 2020;9(6):3766-3775. doi: 10.21037/apm-20-1632 and psychological well-being of Greek hematological cancer patients on chemotherapy. Psychol Health Med 2020;25:201-13.

24. Rodrigues AR, Trufelli DC, Fonseca F, et al. Fatigue in Patients With Advanced Terminal Cancer Correlates With Inflammation, Poor Quality of Life and Sleep, and Anxiety/Depression. Am J Hosp Palliat Care 2016;33:942-7.

(English Language Editor: A. Kassem) 